\title{
New VLA observations of the SNR Puppis A: the radio properties and the correlation with the $\mathrm{X}$-ray emission
}

\author{
G. Castelletti ${ }^{1, \star}$, G. Dubner ${ }^{1, \star \star}$, K. Golap ${ }^{2}$, and W. M. Goss ${ }^{2}$ \\ 1 Instituto de Astronomía y Física del Espacio (IAFE), CC 67, Suc. 28, 1428 Buenos Aires, Argentina \\ e-mail: gcastell@iafe.uba.ar \\ 2 National Radio Astronomy Observatory (NRAO), New Mexico, USA
}

Received 21 February 2006 / Accepted 29 June 2006

\begin{abstract}
Context. High-resolution, high-sensitivity multifrequency radio images of supernova remnants (SNRs) are essential in advancing the understanding of both the global SNR dynamics and particle acceleration mechanisms.

Aims. In this paper we report on a new study of the SNR Puppis A based on VLA observations at $1425 \mathrm{MHz}$; the improvement represents a factor of two in angular resolution and almost ten times in sensitivity compared to the best previous image of Puppis A. This new image is used to compare with re-processed $327 \mathrm{MHz}$ data and ROSAT and Chandra images to investigate morphological and spectral characteristics.

Methods. The observations were carried out with the VLA in the DnC and CnB configurations in 2004. After combining with singledish data from Parkes, an angular resolution of $34^{\prime \prime} \times 16^{\prime \prime}$ and an rms noise of $0.5 \mathrm{mJy}$ beam ${ }^{-1}$, were achieved. Archival VLA data at $327 \mathrm{MHz}$ were also processed. The spectral index distribution was then determined by a direct comparison of the homogenized data at both 327 and $1425 \mathrm{MHz}$. In addition, to identify different spectral components, tomographic spectral analysis was performed. Results. The new $1425 \mathrm{MHz}$ radio image reveals a highly structured border encircling a diffuse, featureless interior. In particular, the northern half of Puppis A displays a complex structure along the periphery, consisting of short arcs resembling "wave-like" features. These are oriented essentially perpendicular to the shock front on the NE side, but are tangential to the shock on the NW side. A remarkable correspondence between such "wave-like" features and spectral changes is observed. On the other hand, the brightest radio features (located to the $\mathrm{E}$ of the SNR and also detected in X-rays) have no counterpart in the spectral index distribution. Based on a uniform compilation of integrated flux densities between 19 and $8400 \mathrm{MHz}$, a global spectral index $\alpha=-0.52 \pm 0.03\left(\mathrm{~S} \propto \gamma^{\alpha}\right)$ has been determined. The new $1425 \mathrm{MHz}$ image of Puppis A was compared with the ROSAT X-ray image of the entire SNR and with the resolved arcsec Chandra image of the bright E region. There is good overall agreement between the radio and high resolution X-ray images. At the arcsec level, however, the agreement of the radio and X-ray images is less striking. A noticeable similarity is found between Puppis A, with its E and W extensions, and analogous morphological features observed in the SNR W50. This suggests that Puppis A could be another case of a SNR shaped by the action of energetic jets.
\end{abstract}

Key words. ISM: individual objects: Puppis A - ISM: supernova remnants - radio continuum: ISM

\section{Introduction}

Sensitive, high-resolution studies of the radio emitting plasma associated with supernova remnants (SNRs) have proven to be an excellent tool for recognizing morphological characteristics, delineating the location of contact discontinuities in the fluid, and spatially resolving filamentary emission that may indicate expansion into non-uniform environments in the interstellar medium (ISM). These high quality radio images are also helpful in addressing questions concerning particle acceleration mechanisms based on determinations of the spatial variations in the radio spectral index. Moreover, the detailed combination of radio and X-ray data not only complements the analysis for particle acceleration, but is also a valuable tool for studying the interaction of the blast wave with inhomogeneities in the surrounding ISM.

Dubner et al. (1991) have presented an earlier VLA image of the SNR Puppis A at $1515 \mathrm{MHz}$ based on observations from a

\footnotetext{
* Post-Doc Fellow of CONICET, Argentina.

$\star \star$ Member of the Carrera del Investigador Científico of CONICET, Argentina.
}

combination of 26 different pointings observed with the VLA ${ }^{1}$ in the DnC array. The angular resolution of this image is $77^{\prime \prime} \times 43^{\prime \prime}$ with a noise of $\sim 3 \mathrm{mJy}^{\text {beam }}{ }^{-1}$. This image shows some agreement between the bright radio features and the X-ray emission detected by Einstein X-ray Observatory. However, the angular resolution and sensitivity of this previous VLA image is not high enough to allow a detailed comparison with improved X-ray images, e.g. that of Petre et al. (1996) obtained using ROSAT, or the detailed high resolution Chandra study of the eastern region carried out by Hwang et al. (2005). Because of advances in both instrumentation and imaging algorithms for the production of sensitive radio mosaics of large regions, a new set of $20 \mathrm{~cm}$ radio observations have been carried out using the VLA.

In this paper, we present a new $1425 \mathrm{MHz}$ image of Puppis A generated from VLA DnC and CnB configuration data sets obtained in 2003 and 2004. The improved sensitivity and resolution have led to the discovery of new morphological features near the outer shock front and at the E side of the SNR shell. From

1 The Very Large Array of the National Radio Astronomy Observatory is a facility of the National Science Foundation operated under cooperative agreement by Associated Universities, Inc. 
the combination of the radio observations at $1425 \mathrm{MHz}$ with reprocessed data at $327 \mathrm{MHz}$ from the VLA archives, we have investigated the spatial spectral index distribution across the remnant. Based on the $1425 \mathrm{MHz}$ image, we have also performed a detailed study of the correlation between radio and X-ray emission, focusing special attention on the complex region to the $\mathrm{E}$.

\subsection{General multiwavelength properties of the SNR Puppis A}

Puppis A is the remnant of a galactic supernova for which it is possible to observe ejecta from the original explosion that occurred $\sim 3700$ yr ago (Winkler et al. 1988). In addition, signatures of the interaction with the circumstellar/interstellar material can be observed. Based on an analysis of both X-ray and optical spectra, Puppis A can be classified as belonging to the small class of galactic oxygen-rich SNRs (Canizares \& Winkler 1981; Winkler \& Kirshner 1985) in which fast moving filaments evidence nuclear processes that occurred in the progenitor star.

The radio emission of Puppis A is of synchrotron origin and takes the form of a highly distorted clumpy shell, with the brightest section along the E border. Optical emission is present as a collection of compact condensations rather than delicate filaments, and shows little relation to the radio features (Milne et al. 1983).

Previous multiwavelength studies of the surroundings of Puppis A suggested the existence of dense interstellar clouds along the E-NE border of the SNR (Petre et al. 1982; Teske \& Petre 1987; Arendt et al. 1990, 1991). This was later supported by atomic and molecular studies of the surrounding interstellar medium; HI and ${ }^{12} \mathrm{CO} \mathrm{J}: 1-0$ line observations have revealed the existence of a chain of interstellar clouds concentrated along the NE periphery of the remnant (Dubner \& Arnal 1988; Reynoso et al. 1995). From these studies, a kinematical distance of $2.2 \pm 0.3 \mathrm{kpc}$ has been derived for Puppis A.

The X-ray remnant consists of non-uniform emission that includes both extended features and knots (Petre et al. 1982), the most conspicuous of which is the bright knot located at the E limb (called by those authors "the bright eastern knot", or "BEK"). This feature coincides with an "indentation" in the radio morphology, suggesting an encounter between the SN shock and an external inhomogeneity. Based on an $\mathrm{OH} 1667 \mathrm{MHz}$ line study, Woermann et al. (2000) suggested that the shock front expanding to the $\mathrm{E}$ has completely engulfed a cloud giving rise to the X-ray knot, leaving no observable unshocked remainder of the cloud. Recently, Hwang et al. (2005) presented Chandra $\mathrm{X}$-ray images and spectral analysis of the region around the BEK. These images reveal details of substructure in this part of Puppis A. The spectral analysis shows a temperature gradient rising behind the shock front, suggesting possible heating by reflected shocks due to the interaction with denser gas.

An unresolved X-ray source has been detected near the centre of Puppis A (RX J0822-4300, Pavlov et al. 1999, and references therein). This source may well be the compact stellar remnant formed in the $\mathrm{SN}$ event. However, no associated pulsar wind nebula has been detected (Gaensler et al. 2000).

\section{Observations}

\subsection{Re-processing of radio continuum data at $327 \mathrm{MHz}$}

$327 \mathrm{MHz}$ data acquired with the VLA in the CnB configuration in 1988 (Dubner et al. 1991) were re-processed. The data were flagged and calibrated using the AIPS software package. Further processing was done in AIPS++. The W-Projection imaging algorithm (Cornwell et al. 2003) was used to correct for the noncoplanarity of the VLA array at this frequency (Cornwell \& Perley 1992). We made use of multiscale clean to deconvolve the image. The deconvolution was done in iterations, with the visibility data being self-calibrated in phase after each iteration.

To recover the very low spatial frequencies that the VLA observations miss, we made use of single-dish data extracted from the $408 \mathrm{MHz}$ All-Sky Continuum Survey (Haslam et al. 1982). We scaled the flux density of the single dish image with a spectral index $\alpha=-0.6$, which we derived from comparing the flux densities at 86 and $408 \mathrm{MHz}$ (Milne et al. 1993). The resultant single-dish image was combined with our interferometric image. The combination is based on a method referred to as "feathering". This method involves Fourier transformation of both the single dish and interferometric data onto identical grids. The single-dish Fourier data is multiplied by the ratio of the Point Spread Function (PSF) volumes of the interferometric image to that of the single dish image. This scaled data is added to the Fourier interferometric data multiplied by a feathering function. The feathering function used in AIPS ++ is $1-\mathrm{FT}\left(\mathrm{PB}_{\mathrm{dish}}\right)$, where $\mathrm{FT}\left(\mathrm{PB}_{\mathrm{dish}}\right)$ denotes the Fourier transform of the primary beam of the single-dish. This combined data is then Fourier transformed back to the image domain.

Although the angular resolution of the final image at $327 \mathrm{MHz}$ is $90^{\prime \prime} \times 45^{\prime \prime}$ at a position angle of $164^{\circ}$, and the rms noise level is $10 \mathrm{mJy}^{\text {beam }^{-1}}$, comparable to the earlier results of Dubner et al. (1991), the newly processed $327 \mathrm{MHz}$ image gives a more reliable representation of Puppis A at all spatial frequencies.

\subsection{New radio continuum observations at $1425 \mathrm{MHz}$}

We observed Puppis A at $1425 \mathrm{MHz}$ using the VLA in the DnC and $\mathrm{CnB}$ configurations. The DnC data were obtained on 2003 January 21, 22, and 23, while the $\mathrm{CnB}$ observations were carried out on 2004 February 4, 5, 7, and 15. The flux density calibrators used were 3C 147 and 3C 286 for the DnC observations and 3C 286 for the $\mathrm{CnB}$ observations. The phase and amplitude calibrator used for all the data observed was the source J0828-375. Due to the large size of this SNR (about 55' in diameter) we used a mosaicking technique with 39 different pointings with a spacing of half of the primary beam width. Each sub-field was observed in two $50 \mathrm{MHz}$ bands centered near 1465 and $1385 \mathrm{MHz}$ in continuum mode. The observations were made in cycles with $28 \mathrm{~s}$ in each pointing of the mosaic. This observing cycle was repeated 9 times in the DnC array and 12 times in the $\mathrm{CnB}$ configuration.

The data were reduced using the AIPS++ software package. The data from each day were calibrated, imaged and selfcalibrated separately to ensure that there were no day-to-day amplitude discrepancies. All the calibrated data sets were then concatenated into a single visibility dataset. We imaged and self-calibrated the combined visibility data in an iterative fashion. The imaging was done using the standard mosaicking technique that jointly deconvolves all the fields, taking into account the primary beam effect at each pointing. The multiscale clean method was applied to deconvolve the image. We used uniformly weighted visibility data for the high resolution image. For the image that is used to compare with the $327 \mathrm{MHz}$ data, the visibility data were tapered in such a fashion that the resulting restoring beam is identical to that of the $327 \mathrm{MHz}$ image.

To recover the large scale structure missed by the interferometer, we used an image from the Parkes Southern Galactic Plane 


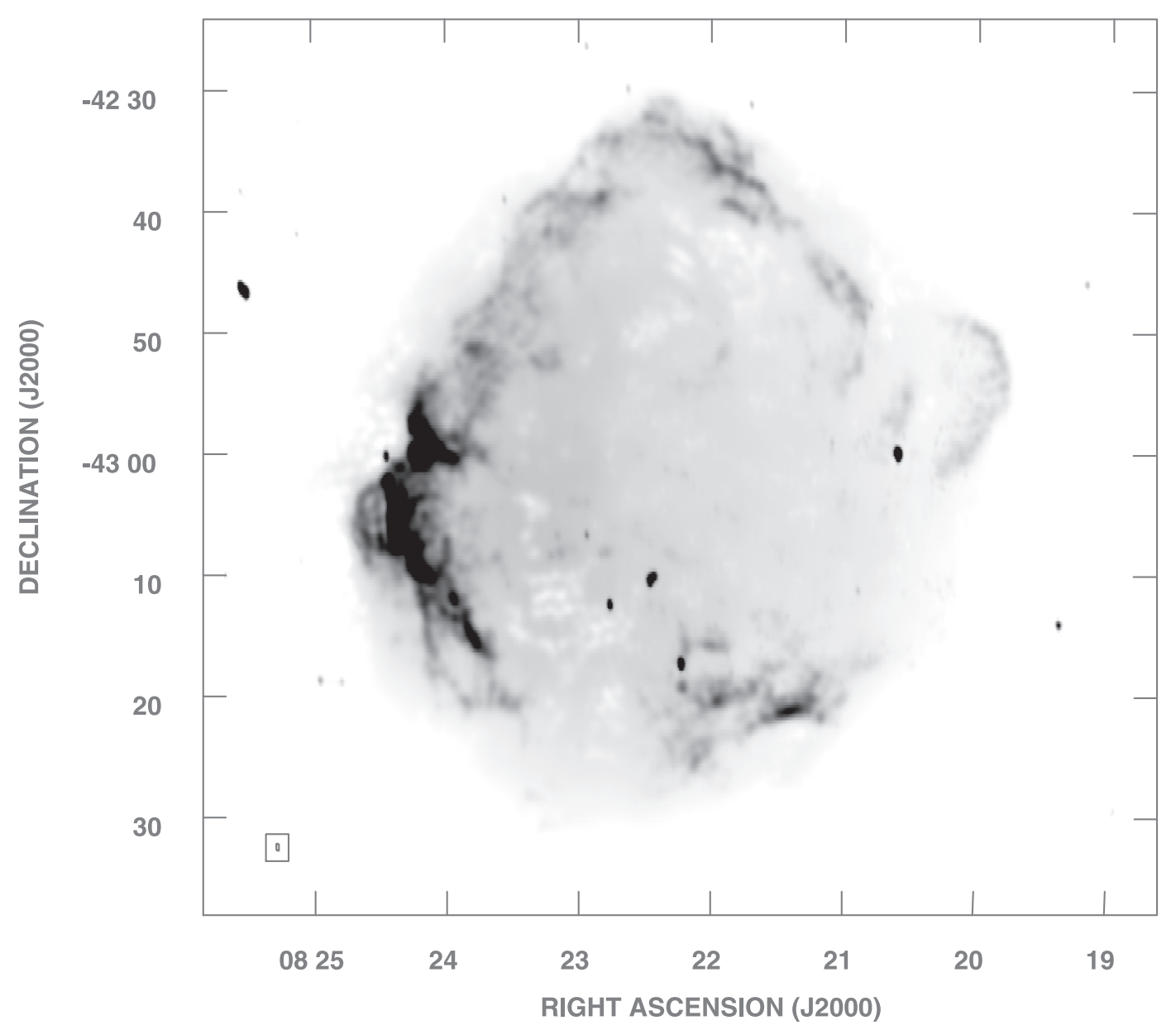

Fig. 1. The SNR Puppis A at $1425 \mathrm{MHz}$. This image was obtained from a combination of 39 different pointings observed with the VLA in the DnC and $\mathrm{CnB}$ configurations in 2004. Single dish observations from the Parkes Southern Galactic Plane Survey (McClure-Griffiths et al. 2001) have been added to the data. The angular resolution is $34^{\prime \prime} \times 16^{\prime \prime}$ (beam shown at lower left), $\mathrm{PA}=-174^{\circ}$ with an rms of $0.5 \mathrm{mJy}^{-1}$ beam ${ }^{-1}$. The brightness range covered by the grayscale is between 3 and $35 \mathrm{mJy}_{\text {beam }}{ }^{-1}$.

Survey (McClure-Griffiths et al. 2001). The single-dish and the interferometric mosaic images were combined using the same feathering technique described above.

The uniform weighting image that was produced has a resolution of $34^{\prime \prime} \times 16^{\prime \prime}$ at a position angle of $-174^{\circ}$, and an rms noise of $0.5 \mathrm{mJy} \mathrm{beam}^{-1}$, representing almost an order of magnitude improvement in sensitivity over the image of Dubner et al. (1991).

\section{Results}

\subsection{The new VLA image of Puppis $A$}

Figure 1 shows the new VLA $1425 \mathrm{MHz}$ image of Puppis A. At this frequency the remnant has the appearance of an asymmetric shell with an average diameter of $\sim 55^{\prime}$ or $35 \mathrm{pc}$ at a distance of $2.2 \mathrm{kpc}$. The intensity of the brightest features along the periphery is spatially variable, ranging from $\sim 8 \mathrm{mJy} \mathrm{beam}^{-1}$ in the $\mathrm{N}$ to $\sim 60 \mathrm{mJy}^{\text {beam }}{ }^{-1}$ towards the E. No sharp boundaries are observed, although the presence of a weak radio halo at a mean level of $3.5 \mathrm{mJy}^{\text {beam }}{ }^{-1}$ is apparent in the original images extending about $5^{\prime}$ ahead of the bright rim. With the addition of short spacing contributions, all the flux density is represented in the image in Fig. 1. This image shows diffuse interior emission at an average level of $8 \mathrm{mJy}^{\text {beam }}{ }^{-1}$.
These new sensitive radio observations show no evidence for any radio counterpart $\left(3 \sigma\right.$ of $\left.1.5 \mathrm{mJy} \mathrm{beam}^{-1}\right)$ to the X-ray point source RX J0822-4300 nor for a pulsar wind nebula, consistent with previous negative results obtained by Gaensler et al. (2000).

Enlargements showing the detailed $1425 \mathrm{MHz}$ total intensity morphology in three selected regions of the Puppis A SNR are shown in Fig. 2 . Due to the high dynamic range $(\approx 1000: 1)$ of the $1425 \mathrm{MHz}$ image, several grayscale ranges are used to display the different relevant regions. Figure $2 \mathrm{a}$ shows the E portion of the SNR shell, Fig. 2b displays the N part of Puppis A, while Fig. 2c shows the locations of the brightest features in the SW edge of the remnant. The new high resolution image reveals that the boundaries of Puppis A are quite structured, in contrast with the smooth interior. The outer border consists of a series of arc-like features, resembling a "wave-like" structure. Such features are more evident in the $\mathrm{N}$ half of Puppis A (Fig. 2b), although some are detected to the SW. We note that along the NW and SW borders, the "wave-like" features are observed tangential to the outer rim, but their orientation changes to approximately radial (i.e perpendicular to the local shock front) along the NE border.

Petre et al. (1982) reported the detection of bright X-ray emission near RA $=08^{\mathrm{h}} 24^{\mathrm{m}}$, Dec $=-43^{\circ} 00^{\prime}(\mathrm{J} 2000)$, the BEK (see Fig. 2a). Intense radio emission is observed coincident with the X-ray emitting area (see Fig. 7 below). In addition, the new high resolution $1425 \mathrm{MHz}$ observations reveal the presence of a 

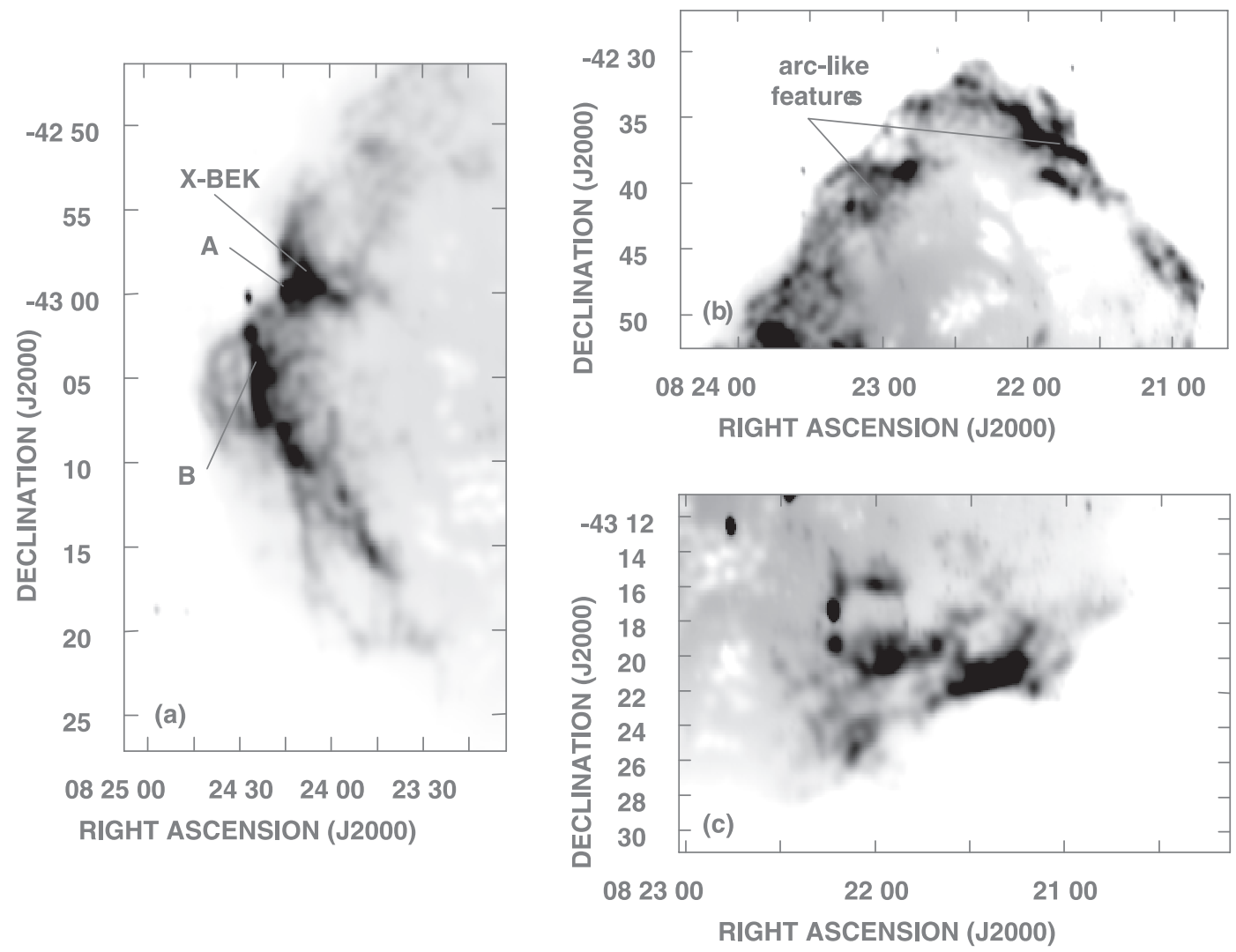

Fig. 2. Enlargements of the new VLA $1425 \mathrm{MHz}$ image. a) E region, containing the brightest features of the remnant. Feature A marks the position of a bright point-like emission region, while B indicates the location of the straight $\mathrm{N}-\mathrm{S}$ filament. The grayscale ranges from 5 to $52 \mathrm{mJy}^{\mathrm{beam}}{ }^{-1}$. b) N part of Puppis A; the irregular outer border is prominent. Note the presence of "wave-like" features almost perpendicular to the shock front on the NE and parallel to it on the NW. The grayscale ranges from 6 to $16 \mathrm{mJy} \mathrm{beam}^{-1}$. c) The $\mathrm{SW}$ region showing the presence of the only bright feature located at the outer boundary of Puppis A. The grayscale varies between 5 and $18 \mathrm{mJy}_{\text {beam }}{ }^{-1}$.

compact (size $\sim 45^{\prime \prime}$ ) feature of enhanced synchrotron emission (feature A in Fig. 2a), exactly at the site where the shock front forms an angle, near RA $=08^{\mathrm{h}} 24^{\mathrm{m}} 18^{\mathrm{s}}$, Dec $=-43^{\circ} 00^{\prime} 00^{\prime \prime}(\mathrm{ob}-$ served in Fig. $2 \mathrm{a}$ as a bright region adjacent to the BEK). To the $\mathrm{S}$ of this feature, the presence of a $\sim 6^{\prime}$ prominent, straight filament (labeled as B in Fig. 2a) is noticed near RA $=08^{\mathrm{h}} 24^{\mathrm{m}} 22^{\mathrm{s}}$, running $\mathrm{N}-\mathrm{S}$ from $\mathrm{Dec}=-43^{\circ} 02^{\prime}$ to $\mathrm{Dec}=-43^{\circ} 08^{\prime}$. To the $\mathrm{E}$ of this filament, a fainter semi-circular protrusion (extending about $6^{\prime}$ ) is observed. The SW region of Puppis A (Fig. 2c) shows the only bright feature located on the edge of the SNR. Short arcs (size $\sim 4^{\prime}$ ), similar to those observed to the NW, are also observed in this region, although in this case they appear displaced by $\sim 8^{\prime}$ from the limb to the interior.

Figure 3 shows the re-processed image at $327 \mathrm{MHz}$ of Puppis A. These data were used with the $1425 \mathrm{MHz}$ image for the spectral analysis discussed in Sect. 4.

\section{The spectral properties}

\subsection{The integrated spectrum}

We have calculated integrated flux densities for Puppis A based on the new images, obtaining: $S_{327 \mathrm{MHz}}=263 \pm 20 \mathrm{Jy}$ and $S_{1425 \mathrm{MHz}}=114 \pm 8 \mathrm{Jy}$. The errors reflect the uncertainties both in the choice of background emission and integration boundaries, in addition to the inherent rms noise of the images.

To construct accurate spectra of individual SNRs it is necessary that all flux density values be brought on to the same absolute flux density scale. Table 1 contains the flux densities between 19 and $8400 \mathrm{MHz}$. All the flux densities for frequencies above $400 \mathrm{MHz}$ are on the Baars et al. (1977) scale. For those below that frequency no scaling was applied. Figure 4 shows the resulting radio spectrum of the Puppis A SNR; the new determinations are plotted as filled circles. The flux density values are adequately fitted by a single power law of slope $\alpha=-0.52 \pm 0.03$, in good agreement with estimates presented by Milne \& Hill (1969) $(\alpha \simeq-0.50)$ and Dubner et al. (1991) $(\alpha \simeq-0.53)$.

\subsection{The spatial spectral distribution}

It is important to discern whether the electron energy spectrum is a global property of this SNR, or if it is locally affected by the neutron star and/or the shock-ISM interaction. Hence, we performed a careful study of the variations in the spectrum as a function of position within the remnant.

The previous study of the spectral index distribution was carried out by Dubner et al. (1991) using VLA observations at 327 and $1515 \mathrm{MHz}$. The distribution of the spectral indices was estimated by integrating flux densities within five rectangular boxes (of different sizes according to the region, varying between approximately 16 and $190 \mathrm{arcmin}^{2}$ ), and comprising the brightest portions of Puppis A. An index as steep as $\alpha \sim-0.7$ was suggested for the E border, while for the other four regions the spectral index was found to be about -0.5 . Using more recent spectral analysis techniques and the new VLA $1425 \mathrm{MHz}$ data, we are able to produce a detailed analysis of the spatial spectral 


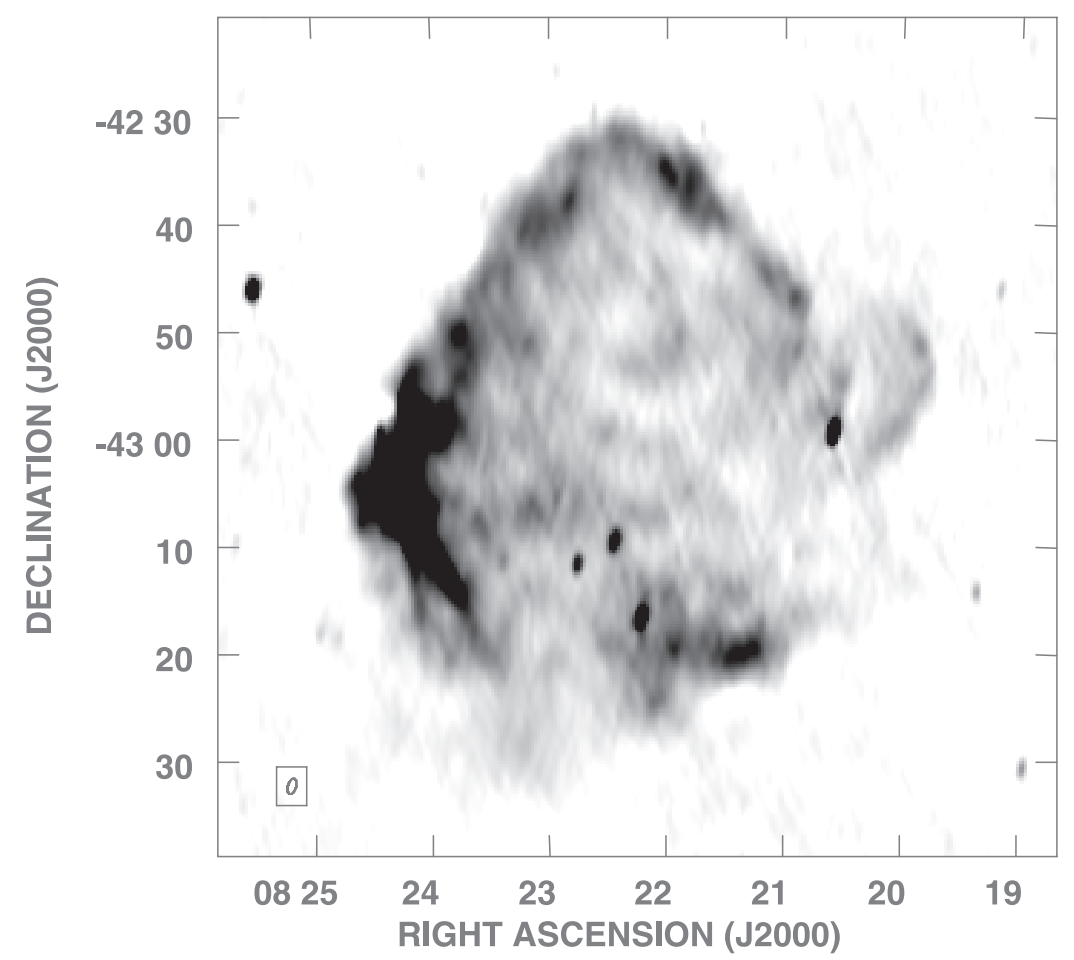

Fig. 3. Radio continuum image of SNR Puppis A at $327 \mathrm{MHz}$ obtained with the VLA in the CnB configuration in 1988 and now re-processed. The interferometric data were combined with single dish data from MPIfR Bonn radiotelescope (at $408 \mathrm{MHz}$, Haslam et al. 1982). The final beam size is $90^{\prime \prime} \times 45^{\prime \prime}$ (shown at lower left) at a position angle of $164^{\circ}$, and the rms noise level is $10 \mathrm{mJy}$ beam ${ }^{-1}$. The grayscale ranges from 35 to $350 \mathrm{mJy}^{\text {beam }}{ }^{-1}$.

Table 1. Integrated flux densities for the SNR Puppis A.

\begin{tabular}{lll}
\hline \hline $\begin{array}{l}\text { Frequency } \\
(\mathrm{MHz})\end{array}$ & $\begin{array}{l}\text { Scaled flux } \\
\text { density (Jy) }\end{array}$ & References \\
\hline 19 & $800 \pm 160^{a}$ & Rishbeth (1958) \\
86 & $690 \pm 100^{a}$ & Mills et al. (1960) \\
327 & $263 \pm 20$ & This work $^{(b)}$ \\
408 & $235 \pm 20$ & Haslam et al. (1982) \\
635 & $180 \pm 29$ & Milne \& Hill (1969) \\
843 & $144 \pm 10$ & Arendt et al. (1990) \\
960 & $130 \pm 12$ & Harris (1962) \\
1410 & $129 \pm 20$ & Milne \& Hill (1969) \\
1425 & $114 \pm 8$ & This work ${ }^{(b)}$ \\
1440 & $166 \pm 17$ & Mathewson et al. (1962) \\
1515 & $118 \pm 10$ & Dubner et al. (1991) \\
2650 & $92 \pm 14$ & Milne \& Hill (1969) \\
2700 & $78 \pm 12$ & Milne (1971) \\
4750 & $59 \pm 5$ & Milne et al. (1993) \\
5000 & $67 \pm 7$ & Milne \& Hill (1969) \\
5000 & $61 \pm 7$ & Milne (1971) \\
8400 & $38 \pm 4$ & Milne et al. (1993) \\
\hline
\end{tabular}

${ }^{a}$ No correction to Baars et al. (1977) scale was applied.

${ }^{b}$ Flux density scale from VLA Calibrator Manual,

http: /www.aoc.nrao.edu/ gtaylor/calib.html

transition from the bright features to the smooth weaker interior of this SNR.

To accurately determine the spectral index distribution from the comparison of two interferometric images at different frequencies, it is necessary to ensure that the corresponding images are matched in uv coverage. As mentioned in Sect. 2.2, the data at $1425 \mathrm{MHz}$ were processed by applying appropiate weights to the visibilities to obtain an image with the same beam size and beam shape as the $327 \mathrm{MHz}$ image. Also, in order to avoid any

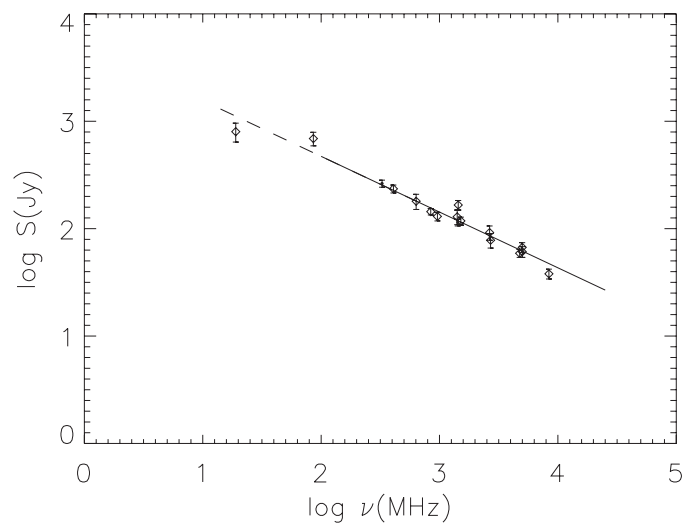

Fig. 4. Radio spectrum of Puppis A from the scaled flux density values listed in Table 1. The data from this paper are shown by filled circles (327 and $1425 \mathrm{MHz}$ ). The linear fit to the values of flux density indicates a global spectral index of $-0.52 \pm 0.03$. The dashed line shows the linear fit extrapolated to 19 and $86 \mathrm{MHz}$, at which frequencies no correction has been applied to bring the measured values to the Baars et al. (1977) scale.

positional offsets, the images were aligned and interpolated to identical projections (field center, pixel separation, etc.). In addition, we checked for possible flux density calibration bias. That is, an extrapolation back to zero flux density at one frequency should correspond to zero flux density at the other frequency, otherwise there is a zero-point offset that must be corrected. We have checked the 327 and $1425 \mathrm{MHz}$ images, finding only an insignificant offset of $0.1 \mathrm{mJy}^{\text {beam }}{ }^{-1}$ between frequency pairs. Therefore, no further correction was applied.

Based on these images, we carried out the spatial spectral analysis. This was done using two different methods in order to 


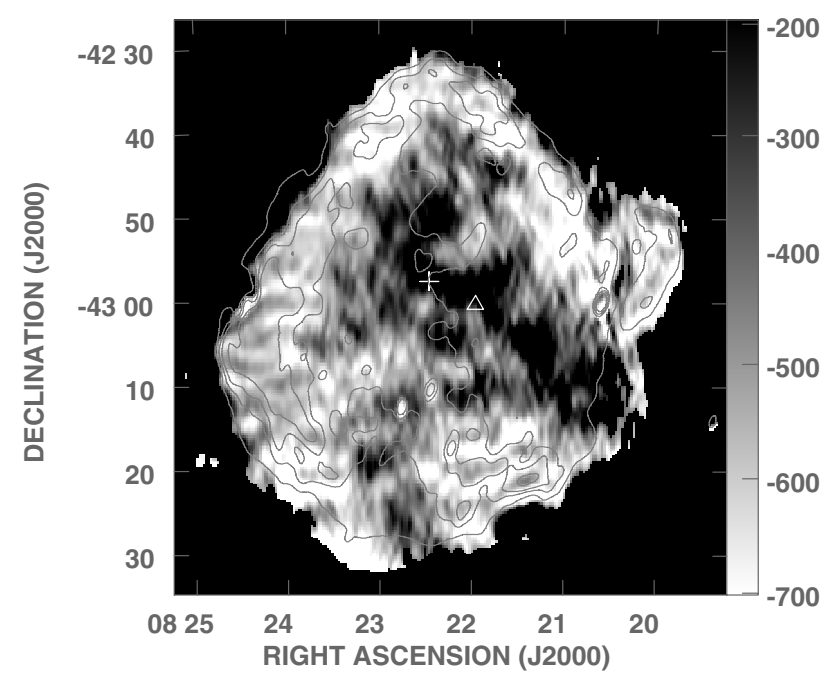

Fig. 5. Grayscale image of the spectral index distribution across the SNR Puppis A as derived between 327 and $1425 \mathrm{MHz}$. The wedge displays the spectral index value $(\times 1000)$. The $1425 \mathrm{MHz}$ intensity contours plotted for reference are 36, 60, 90 and $160 \mathrm{mJy} \mathrm{beam}^{-1}$. A cutoff at $5 \sigma$ was applied at both frequencies. The plus sign indicates the geometrical center of the SN explosion, while the triangle marks the position of the neutron star RX J0822-4300.

confirm that the observed spectral features are real, independent of any possible contamination from the databases used for the comparison.

In Fig. 5 we show the spectral index distribution of Puppis A as obtained from the direct comparison of the matched images at 327 and $1425 \mathrm{MHz}$ after clipping at the $5 \sigma$ level for both frequencies. We find that the spectral index varies across the SNR approximately between $\alpha \sim-0.8$ and -0.2 . In this grayscale representation, darker means flatter spectrum. It is interesting to note that the feature with the flattest spectrum (seen as a black band running from $\mathrm{NE}$ to $\mathrm{SW}$ in the interior of Puppis A) includes the location of the neutron star RX J08224300 and of the geometrical center of the explosion as derived from optical observations by Winkler et al. (1988) (shown in Fig. 5 as a triangle and a plus sign respectively). These positions are known with an accuracy of tenths of an arcsec for RX J0822-4300 and $\sim 1$ arcmin for the proposed center of the explosion. We can confidently assume that this spectral feature is real since both images have recovered all flux density.

The present spectral map shows that along most of the periphery the spectrum is clearly steep (steeper than $\alpha=-0.7$ ). To the $\mathrm{W}$, the ear-like feature shows a combination of different structures with variable spectral indices.

Curiously the spectral pattern observed near the bright eastern radio features (approximately near RA $\sim 08^{\mathrm{h}} 24^{\mathrm{m}}$, from Dec $\sim-42^{\circ} 55^{\prime}$ to $\sim-43^{\circ} 10^{\prime}$ ) does not correlate with the morphology observed in total power (see Fig. 1). Although it is somewhat flatter than the rest of the shell, it is apparent that the spectrum varies following a pattern of parallel fringes that alternate steeper and flatter spectral indices.

In order to test the spectral index distribution determined above, we also constructed a gallery of tomographic maps. Spatial tomography between two images is a technique developed by Katz-Stone \& Rudnick (1997a), based on the use of a test spectral index $\alpha_{\mathrm{t}}$. A tomographic map is calculated by scaling the brightness in the higher frequency image $\left(S_{1425 \mathrm{MHz}}\right.$, in our case) by the test spectral index, and subtracting this scaled image from the lower frequency image $\left(S_{327 \mathrm{MHz}}\right)$. Then, a tomographic gallery is obtained by calculating $S_{\mathrm{t}}=S_{327 \mathrm{MHz}}-$ $\left(\frac{327}{1425}\right)^{\alpha_{\mathrm{t}}} S_{1425 \mathrm{MHz}}$, stepping through a range of spectral indices $\alpha_{\mathrm{t}}$. Features which have a spectral index identical to the test value will vanish in the tomographic map. Spatial components that have different spectral indices will appear as positive or negative features depending upon whether the spectrum is steeper or flatter than the assumed test value. This method has been succesfully applied to analyze spatial spectral variations in the SNRs of Tycho (Katz-Stone et al. 2000), Kepler (DeLaney et al. 2002), G292.0+1.8 (Gaensler \& Wallace 2003) and in the study of the radio galaxies 3C 67, 3C 190 and 3C 449 (Katz-Stone \& Rudnick $1997 \mathrm{a}, \mathrm{b})$. The technique is particularly useful to disentagle components with different spectra that can overlap along the line of sight. Also, when the goal is to locate small-scale spectral variations, this method can provide a more accurate picture than the simple comparison between images at different frequencies.

In Fig. 6 we show the resulting tomographic images for four test values: $\alpha_{\mathrm{t}}=-0.2$ (Fig. 6a), -0.4 (Fig. 6b), -0.6 (Fig. 6c), and -0.8 (Fig. 6d). In these maps the spatial components appear as positive (light) or negative (dark) features for a spectrum steeper or flatter than the assumed test value respectively. The point sources overlapping the remnant are always seen as positive residuals in the tomographic images (white spots) as they have spectral indices steeper than -0.8 , thus supporting their extragalactic nature, as already noted by Milne et al. (1983) and Dubner et al. (1991).

The most interesting tomographic images are those obtained from the comparison with $\alpha_{\mathrm{t}}=-0.4$ (Fig. 6b) and with $\alpha_{\mathrm{t}}=$ -0.6 (Fig. 6c), where, as noticed before, a notable striation is evident along the periphery. It is worth noting that this spectral pattern formed by short fringes with $\alpha$ alternatively steeper and flatter than the background, mimics the "wave-like" morphology noticed in the total power image along the NE, NW and S borders. That is, the spectral components appear almost parallel to the outer boundary to the NW and SW, and transverse to it along the E side.

The newly identified point-like feature to the E of Puppis A (feature A in Fig. 2a), has a spectrum steeper than -0.8 (i.e. it appears as a positive residual in all tomographic images). We thus conclude that, in all probability, it is an extragalactic object.

The central band seen in Fig. 5 disappears against the local background in the tomographic image traced with $\alpha_{\mathrm{t}}=-0.2$ (Fig. 6a), thus confirming its very flat spectrum.

The grayscale corresponding to the $\alpha_{\mathrm{t}}=-0.8$ image (Fig. 6d) reveals that the spectral distribution in Puppis A has practically no components steeper than this value.

\section{Comparison between radio and $\mathrm{X}$-ray emission}

The X-ray properties of Puppis A have been investigated using the Einstein, ASCA and ROSAT telescopes (Pavlov et al. 1999, and references therein). The highest resolution X-ray image of the entire source was obtained with ROSAT (HRI) in the energy band from 0.1 to $2 \mathrm{keV}$ (Petre et al. 1996).

In Fig. 7 we present a false color image showing the spatial comparison between the radio image at $1425 \mathrm{MHz}$ (in red) and the soft X-ray emission (in green) for the entire remnant. For the first time a comparison with a spatial resolution $<30^{\prime \prime}$ is possible. Features where both spectral bands overlap are shown in yellow.

The X-ray emission associated with Puppis A has been interpreted as resulting from several thermal components (Berthiaume et al. 1994). The $\mathrm{N}$ half of Puppis A is clearly the brighter and more complex in X-rays. A network of small 

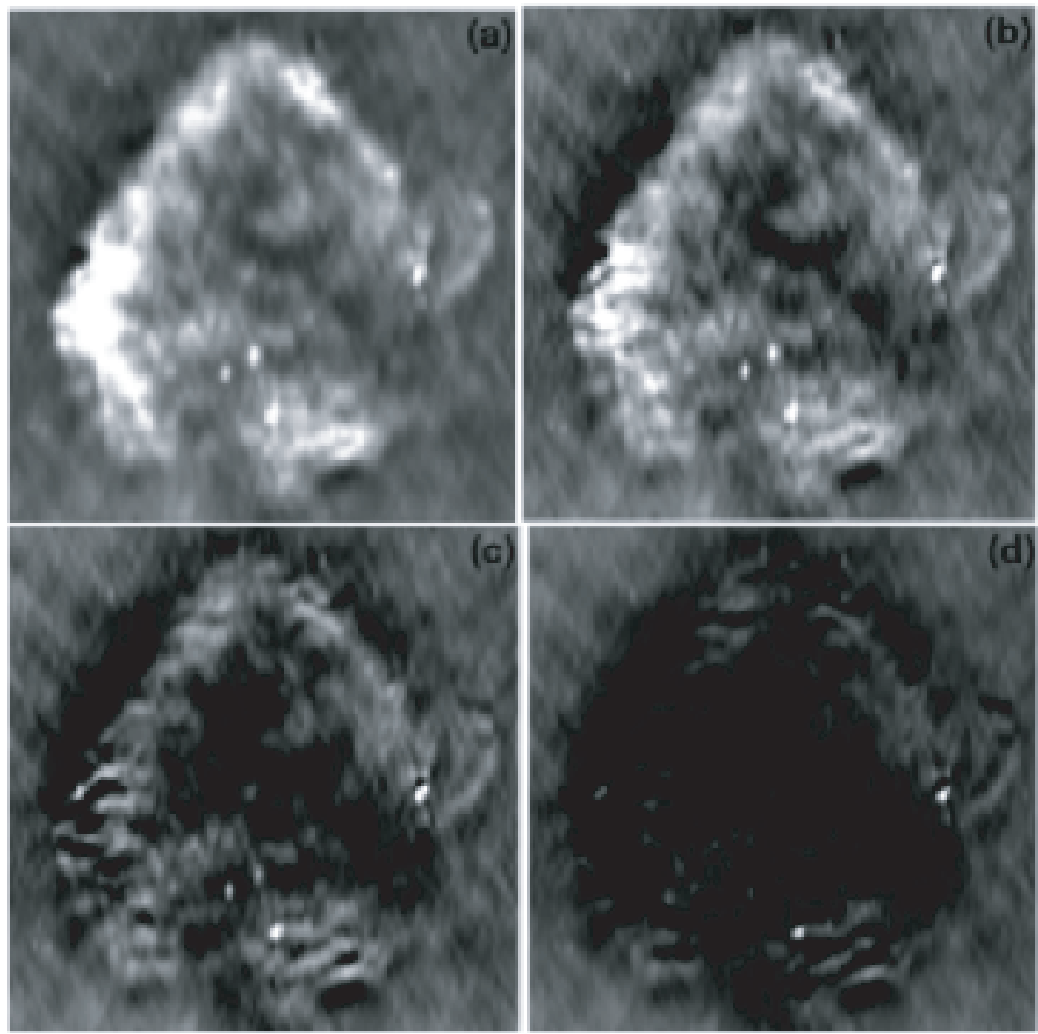

Fig. 6. Series of tomographic images for the SNR Puppis A constructed from data at 327 and $1425 \mathrm{MHz}$, which were matched in uv coverage. a) $\alpha_{\mathrm{t}}=-0.2$; b) $\alpha_{\mathrm{t}}=-0.4$; c) $\alpha_{\mathrm{t}}=-0.6$; and d) $\alpha_{\mathrm{t}}=-0.8$. Bright areas have radio spectral indices steeper than the assumed $\alpha_{\mathrm{t}}$ and dark areas imply flatter spectral indices. The same grayscale is used to display the four images.

scale structures that include filaments, short arcs and knots are, in general, poorly correlated with radio features. The brightest features are preferentially located to the E although the N-NW rim is also well delineated by bright X-ray filaments. Figure 7 demonstrates that the most striking radio/X-ray correspondence occurs predominantly along the E limb of the remnant. Three features are well matched: the BEK, the curved filament near RA $=08^{\mathrm{h}} 23^{\mathrm{m}} 20^{\mathrm{s}}$, Dec $=-43^{\circ} 02^{\prime}$ (see below) and the indentation around RA $\sim 08^{\mathrm{h}} 24^{\mathrm{m}} 30^{\mathrm{s}}$, Dec $\sim-43^{\circ} 00^{\prime}$. In contrast, little radio/X-ray agreement is observed in the SE sector of Puppis A; the radio emission extends over $5^{\prime}$ beyond the $\mathrm{X}$-ray limb. Moreover, the protrusion ahead of the bright, straight $\mathrm{N}-\mathrm{S}$ radio filament (feature $\mathrm{B}$ in Fig. 2a) lacks a counterpart in X-rays. A similar radio/X-ray comparison cannot be carried out to the SW of Puppis A due to a missing ROSAT pointing in this direction (see the ROSAT archive); thus the reconstruction of the image is likely incomplete in this region. Towards the NW border of Puppis A the X-ray emission accompanies the "wavelike" morphology revealed in the radio, with the same orientation parallel to the shock front. The dense X-ray knot located immediately behind the NW shock front (the "bright northern knot”, BNK, RA $\sim 08^{\mathrm{h}} 22^{\mathrm{m}}$, Dec $\left.\sim-42^{\circ} 37^{\prime}\right)$ is prominent in both spectral domains. Petre et al. (1982) have suggested that this knot originated as a result of the interaction with a dense neutral cloudlet, similiar to the BEK. In the radio domain, the spectrum of this knot between 327 and $1425 \mathrm{MHz}$ has a spectral index $\alpha \sim-0.5$ (see Sect. 4.2), similar to the global spectral index of this SNR. A careful spectral study of this clump in X-rays would help towards understanding its origin.

Hwang et al. (2005) have used the Chandra X-ray telescope to resolve the fine structure around the BEK over a field of view of $8^{\prime} \times 8^{\prime}$. These authors describe the inner structure in this part of the remnant as consisting of: (1) a bright compact knot, (2) a curved vertical feature (named the "bar") about 1' W behind the shock, and (3) a smaller bright cloud (the "cap") separated from the "bar" by faint diffuse emission. Nomenclature for these $\mathrm{X}$-ray features is contained in Fig. 8.

To study the relation between the X-rays and the radio components in the Puppis A SNR, in Fig. 8 we show a direct comparison of the new $1425 \mathrm{MHz}$ VLA image with the Chandra $\mathrm{X}$-ray image obtained from the combination of the soft, medium, and hard X-rays (note that the Chandra image has an angular resolution about 5 times finer than the radio image). Figure 8 reveals that the $\mathrm{X}$-ray emitting plasma does not coincide with radio features at a detailed level. However, on a larger scale reasonable agreement is observed. The radio emission is compressed in the indented eastern X-ray boundary, confirming that the shock wave is wrapping around an obstacle in the neutral ISM as was earlier proposed by Hwang et al. (2005). A local radio maximum matches the BEK, as shown in Fig. 8. In this portion of the SNR, this feature is not the brightest feature in radio as is the case for $\mathrm{X}$-rays. The most intense radio emission is found to the $\mathrm{E}$ of the X-ray "bar" (around RA $=08^{\mathrm{h}} 24^{\mathrm{m}} 14^{\mathrm{s}}$, Dec $=-42^{\circ} 59^{\prime} 49^{\prime \prime}$ ), where it correlates with a secondary X-ray peak (see Fig. 8). No conspicuous radio feature can be associated with the "cap". The region of faint diffuse X-ray emission between the "bar" and the "cap" is a region of low surface brightness radio emission.

In summary, based on the present comparison only moderate agreement between radio and X-ray emission has been observed at a detailed level. This conclusion is not surprising since the X-rays can be associated with thermal radiation from a hot plasma, while the radio continuum arises via synchrotron emission from relativistic electrons. 


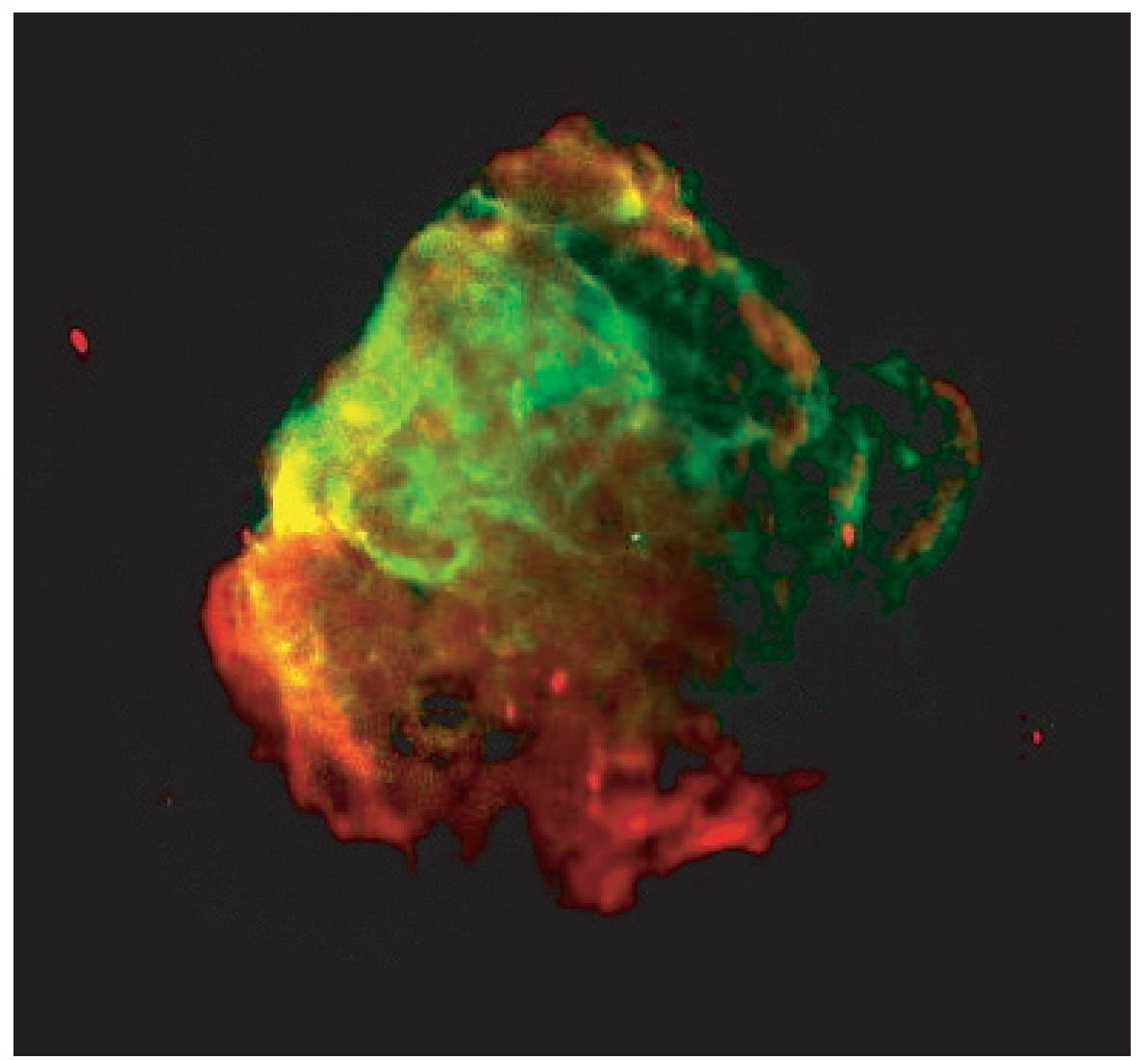

Fig. 7. A high resolution X-ray/radio comparison of the Puppis A SNR. The green image corresponds to soft X-ray emission from ROSAT in the $0.1-2 \mathrm{keV}$ range (Petre et al. 1996), while in red the $1425 \mathrm{MHz}$ radio emission is shown. The yellow regions are areas where emission in both spectral ranges overlap. The X-ray image is smoothed to the resolution of the radio image, $34^{\prime \prime} \times 16^{\prime \prime}, \mathrm{PA}=-174^{\circ}$.

\section{Is Puppis A a new case of a Galactic SNR shaped by energetic jets?}

As noticed before, the general shell-like morphology of Puppis A appears distorted to the E by the protrusion near RA $08^{\mathrm{h}} 24^{\mathrm{m}} 30^{\mathrm{s}}$, Dec $\sim-43^{\circ} 06^{\prime}$ and to the $\mathrm{W}$ by the "ear" around $\mathrm{RA} \sim 08^{\mathrm{h}} 20^{\mathrm{m}} 00^{\mathrm{s}}$, Dec $\sim-42^{\circ} 54^{\prime}$ (Figs. 1, 2a and 7). If we trace two intersecting lines oriented about a position angle of $\sim 100^{\circ}$ (from $\mathrm{N}$ through $\mathrm{E}$ ), with a semi-opening angle of $\sim 15^{\circ}$, crossing at the position of the neutron star candidate RX J0822-4300, the appearance of Puppis A strikingly resembles that of the SNR W50. The shell of W50 is distorted by the precessing jets that originate in the central compact source SS433, and Fig. 9 illustrates the similarity between the two SNRs. In Fig. 9 (left) we have plotted a sketch of the hypothetical "cones" overlapping the new radio image of Puppis $\mathrm{A}$, while on the right side an image of the SNR W50 at $1.4 \mathrm{GHz}$ (from Dubner et al. 1998) is shown with the precession cones of $\sim 20^{\circ}$ semi-opening angle delimited by the intersecting white lines. In the case of W50, the elongated shape has been interpreted as being due to the action of the two oppositely directed relativistic jets injected by the X-ray binary SS433. The present comparison shows that not only the general appearance with symmetrical lateral extension is similar for both SNRs, but that the analogy also extends to particular features. For example, the bright straight N-S filament in Puppis A (named B in Fig. 2a) looks very similar to the straight radio filament that stands out near the E extremity of W50, and which is thought to be the radio "hot-spot" produced by the interaction of the relativistic jet from SS433 with the ISM (Safi-Harb \& Ögelman 1997). Beyond these bright, straight filaments, diffuse radio emission is observed in both cases.
The compact X-ray source RX J0822-4300 in Puppis A has been proposed to be the compact stellar remnant formed in the SN explosion (Pavlov et al. 1999). It is a radio-quiet neutron star belonging to the class of "Central Compact Objects" (CCOs) in SNRs whose nature remains unclear (Pavlov et al. 2002). Based on detailed HI observations carried out in the direction of RX J0822-4300, Reynoso et al. (2003) suggested that this CCO could be ejecting two oppositely directed jets that are creating a bi-lobed cavity in the HI. This elongated hole is about $15^{\prime}$ long, oriented in the NE-SW direction, with a position angle of about $60^{\circ}$. Both the X-ray point source and the position of the geometrical center of the explosion (suggested to be at RA $\sim 08^{\mathrm{h}} 22^{\mathrm{m}} 27.5$, Dec $\sim-42^{\circ} 57^{\prime} 29^{\prime \prime}$, Winkler et al. 1988) lie within the HI hole. Thus, the possible HI signature of jets is not exactly aligned with the direction of the hypothetical cones that connect the large scale $\mathrm{E}$ and $\mathrm{W}$ extensions. However, it cannot be discounted that the HI feature is related to the path of the neutron star from the center of explosion to its present position, while the large scale cones are likely to be associated with the persistent action of jets on the surrounding SNR shell.

In the X-ray domain, no conspicuous feature is apparent in the immediate vicinity of RX J0822-4300 (see Figs. 7 and 8 left). The first bright X-ray features within the hypothetical cones are the thin filament to the E near RA $\sim 08^{\mathrm{h}} 24^{\mathrm{m}} 15^{\mathrm{s}}$, Dec $\sim-43^{\circ} 05^{\prime}$ and to the west near RA $\sim 08^{\mathrm{h}} 20^{\mathrm{m}} 30^{\mathrm{s}}$, Dec $\sim-43^{\circ} 00^{\prime}$ (see Fig. 8 left for a representation of the X-ray emission in equatorial coordinates). At the assumed distance of $2.2 \mathrm{kpc}$, these features occur at about $\sim 32 \mathrm{pc} E$ and $\sim 22 \mathrm{pc}$ W of the central point X-ray source. In the case of W50, the X-ray lobes are symetrically displaced E and W of SS433 starting at about $15^{\prime}$ from the center (approximately $17 \mathrm{pc}$ at the distance of W50) and have a knotty 

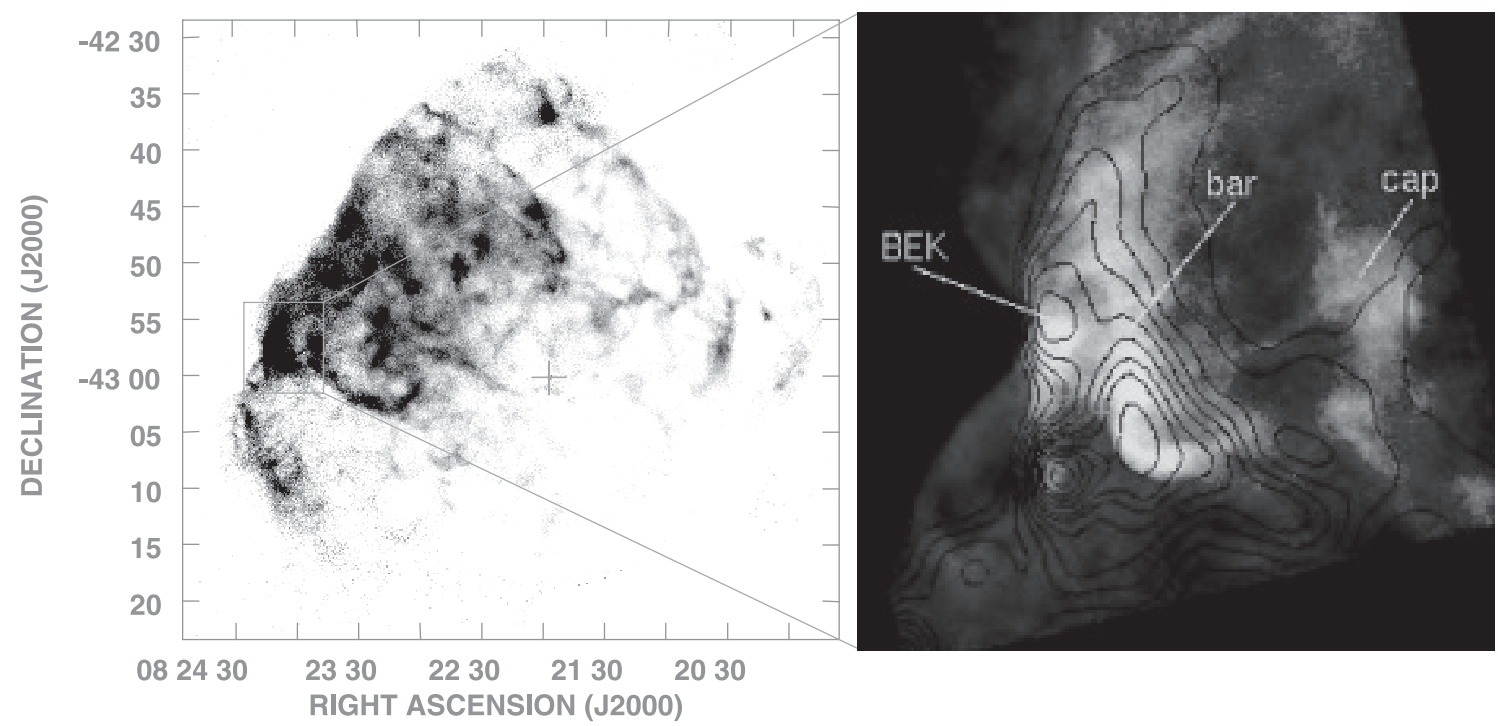

Fig. 8. (Left) ROSAT HRI mosaic of the Puppis A SNR. The box indicates the region of the E limb observed with Chandra (Hwang et al. 2005). The plus (+) symbol shows the position of the compact source RX J0822-4300. (Right) The grayscale representation combines soft ( 0.4-0.7 keV), medium $(\sim 0.7-1.2 \mathrm{keV})$, and hard band $(>1.2 \mathrm{keV})$ images around the bright eastern knot, while the superposed contours trace the radio synchrotron emission at $1425 \mathrm{MHz}$ at $18,23,29,36,43,50,56,61,68,74,80$, and 87 mJy beam $^{-1}$.
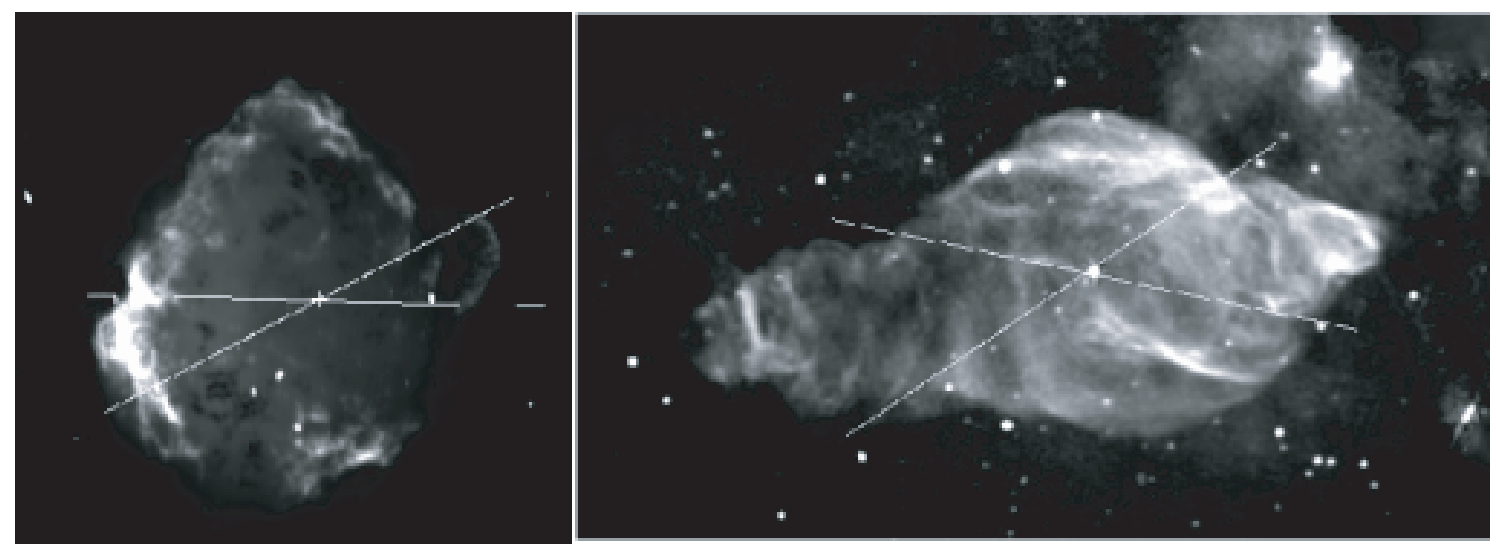

Fig. 9. Comparison of the SNR Puppis A (left) with the SNR W50 that contains the central X-ray binary SS433 (right, from Dubner et al. 1998). In Puppis A, the white cross shows the location of the X-ray point source RX J0822-4300, a radio-quiet neutron star proposed to be the compact remnant of Puppis A explosion. The white intersecting lines delimit the "cones" proposed to be associated with Puppis A in analogy with the case of W50. In the image of the SNR W50, the white lines trace the volume defined by the oppositely directed, precessing, relativistic jets.

structure with enhanced emission at about $35^{\prime}$ (i.e. about $40 \mathrm{pc}$ ) from SS433 (Safi-Harb \& Ögelman 1997). The displacement of the X-ray lobes in W50 has been interpreted by Safi-Harb \& Ögelman (1997) as due to the expansion of beams in a low density ISM.

A careful study of the X-ray features in the interior of Puppis A is highly needed to understand the interaction of the central neutron star with the surrounding SNR and to answer the question as to whether Puppis A is another example of a Galactic SNR distorted by the action of symmetric energetic jets.

\section{Conclusions}

In the current work we have presented a new image of the SNR Puppis A at $1425 \mathrm{MHz}$. This image has revealed with superb detail the presence of short filaments along most of the distorted periphery of Puppis A. In particular, in the northern half of Puppis A the radio emission near the limb resembles a "wavelike" structure, oriented parallel to the shock front along the NW but almost perpendicular to it towards the brighter NE limb. Such a pattern is replicated in the spatial spectral distribution, where fringes with analogous orientation alternate between flat and steep spectra. We propose that the peripheral filaments are a manifestation of Rayleigh-Taylor (R-T) instabilities that are distorting the interface between the ejecta and the ambient material. As R-T instabilities develop, they stretch and compress the magnetic field (Jun \& Norman 1996). This produces magnetic field amplification in the ambient medium close to the interface, which in turn enhances the synchrotron emission. In our model, the R-T fingers observed to the NE are radial, while to the NW they are tangential suggesting that along this border the shock is probably in a more advanced evolutionary stage. This could be related to the possibility that to the NE the shock front is expanding in a denser ISM. To confirm this hypothesis, it is important to investigate the magnetic field orientation in the region. A detailed study of polarization vectors at high angular resolution is planned for the near future. 
Similar short, curved filaments are also observed in the young SNRs of Tycho and Cas A, both in the radio and in X-rays, although in these cases a weaker plateau and a thin brighter outer rim is observed ahead of the filaments (Reynoso et al. 1997; Hwang et al. 2004). In Puppis A, a faint plateau can be clearly seen around almost all the periphery, but no sharp outer border, presumably associated with the foward shock, is detected in this intermediate-age SNR.

The most conspicuous radio features are situated at the E border, at the site where the shock front has engulfed a dense ISM cloud. Interestingly enough, the radio counterpart of the BEK and the narrow straight filament located just south of it do not appear as spectrally distinct features. If these synchrotron maxima are the result of the interaction with external clouds, then we are led to the conclusion that this condition is not reflected in the radio spectrum. The spectral map of Puppis A does not show diferences between the $\mathrm{E}$ and the $\mathrm{W}$ sides in spite of the gradient in density in the ISM (Petre et al. 1982). We can therefore conclude that in this case the shocks are probably still nonradiative and the compression is not very high. This contention is supported by the lack of optical filaments in the boundaries and the generally poor radio/optical correlation (Dubner et al. 1991).

Another spectral peculiarity in Puppis A is the fact that the spectrum steepens in general from the interior to the periphery. In the first-order Fermi, non-relativistic, test particle limit, stronger shocks yield flatter indices up to the $\alpha=-0.5$ limit. Therefore, based on this acceleration model, higher emissivity regions should have flatter spectrum, while steep spectra are expected in the more diffuse emission regions (see for example Anderson \& Rudnick 1993), exactly the opposite of what we find in Puppis A. This result is confirmed through the tomographic analysis of the spectrum. It is interesting to note that the region of Puppis A with the flattest spectrum is a narrow band towards the interior, like a channel that connects the position of the neutron star RX J0822-4300 with the presumed geometrical center of explosion. The continuous injection of relativistic particles by the neutron star could provide an explanation for this spectral characteristic.

Based on a compilation of integrated flux densities over a wide spectral range, we have recalculated the global non-thermal radio spectrum of the SNR Puppis A. The fit produces a spectral index of $\alpha=-0.52 \pm 0.03$ for the whole SNR.

We have also used the new radio image to perform the first accurate radio/X-ray comparison for Puppis A. In X-rays, Puppis A is bright on the E half, and the emission seen by ROSAT between 0.1 and $2 \mathrm{keV}$ is very structured. The new radio image, although much more sensitive than previous ones, does not show detailed correlation with the thermal plasma imaged in X-rays, except for the bright $\mathrm{E}$ features. Even there, the matching is not accurate when the radio emission is compared with the high resolution Chandra image. The only remarkable coincidence revealed by the new results occur along the N-NW boundaries, where the "wave-like" radio features near the shock front have their exact counterpart in the X-rays. A detailed X-ray study along the NW side, including the BNK, would be valuable. To the S-SW of Puppis A, we were unable to carry out a similar comparison because a ROSAT pointing is missing there.

On the other hand, based on the new observations, we have confirmed that down to $\sim 1.5 \mathrm{mJy}_{\text {beam }}^{-1}$ neither a radio counterpart to the neutron star RX J0822-4300, nor a surrounding pulsar wind nebula, is observed.
Finally, based on the radio images and their comparison with the X-ray emission, a striking similarity between Puppis A and the SNR W50, which harbors the Galactic microquasar SS433 in its interior, is seen. This suggests that Puppis A is a candidate for being another SNR shaped by internal jets, probably at an earlier evolutionary stage. Detailed central X-ray images and spectral studies would be of great help to test this suggestion and to understand the nature of the central X-ray compact object.

Acknowledgements. We would like to thank Naomi McClure-Griffiths for providing us with the $1.4 \mathrm{GHz}$ data from the Parkes Southern Galactic Plane Survey. We are grateful to the referee for all his/her suggestions which certainly has helped to make this a better paper. This work has been supported by Argentina grants ANPCYT-PICT 04-14018, UBACYT A055/04, ANPCYTPICT 03-11235 and PIP-CONICET 6433.

\section{References}

Anderson, M. C., \& Rudnick, L. 1993, ApJ, 408, 514

Arendt, R. G., Dwek, E., \& Petre, R. 1991, ApJ, 368, 474

Arendt, R. G., Dwek, E., Petre, R., et al. 1990, ApJ, 350, 266

Baars, J. W. M., Genzel, R., Pauliny-Toth, I. I. K., \& Witzel, A. 1977, A\&A, 61, 99

Berthiaume, G. D., Burrows, D. N., Garmire, G. P., \& Nousek, J. A. 1994, ApJ, 425,132

Canizares, C. R., \& Winkler, P. F. 1981, ApJ, 246, L33

Cornwell, T. J., Golap, K., \& Bhatnagar, S. 2003, EVLA, memo, 67, 1

Cornwell, T. J., \& Perley, R. A. 1992, A\&A, 261, 353

DeLaney, T., Koralesky, B., Rudnick, L., \& Dickel, J. R. 2002, ApJ, 580, 914

Dubner, G. M., \& Arnal, E. M. 1988, A\&AS, 75, 363

Dubner, G. M., Braun, R., Winkler, P. F., \& Goss, W. M. 1991, AJ, 101, 1466

Dubner, G. M., Holdaway, M., Goss, W. M., \& Mirabel, I. F. 1998, AJ, 116, 1842

Gaensler, B. M., Bock, D. C.-J., \& Stappers, B. W. 2000, ApJ, 537, L35

Gaensler, B. M., \& Wallace, B. J. 2003, ApJ, 594, 326

Harris, D. E. 1962, ApJ, 135, 661

Haslam, C. G. T., Stoffel, H., Salter, C. J., \& Wilson, W. E. 1982, A\&AS, 47, 1

Hwang, U., Flanagan, K. A., \& Petre, R. 2005, ApJ, 635, 355

Hwang, U., Laming, J. M., Badenes, C., et al. 2004, ApJ, 615, L117

Jun, B.-I., \& Norman, M. L. 1996, ApJ, 472, 245

Katz-Stone, D. M., Kassim, N. E., Lazio, T. J. W., \& O’Donnell, R. 2000, ApJ, 529,453

Katz-Stone, D. M., \& Rudnick, L. 1997a, ApJ, 479, 258

Katz-Stone, D. M., \& Rudnick, L. 1997b, ApJ, 488, 146

Mathewson, D. S., Healey, J. R., \& Rome, J. M. 1962, Aust. J. Phys., 15, 354

McClure-Griffiths, N. M., Green, A. J., Dickey, J. M., et al. 2001, ApJ, 551, 394

Mills, B. Y., Slee, O. B., \& Hill, E. R. 1960, Aust. J. Phys., 13, 676

Milne, D. K. 1971, Aust. J. Phys., 24, 429

Milne, D. K., Goss, W. M., \& Danziger, I. J. 1983, MNRAS, 204, 237

Milne, D. K., \& Hill, E. R. 1969, Aust. J. Phys., 22, 211

Milne, D. K., Stewart, R. T., \& Haynes, R. F. 1993, MNRAS, 261, 366

Pavlov, G. G., Sanwal, D., Garmire, G. P., \& Zavlin, V. E. 2002, in Neutron Stars in Supernova Remnants, ed. P. O. Slane, \& B. M. Gaensler, ASP Conf. Ser., 271, 247

Pavlov, G. G., Zavlin, V. E., \& Trümper, J. 1999, ApJ, 511, L45

Petre, R., Becker, C. M., \& Winkler, P. F. 1996, ApJ, 465, L43

Petre, R., Kriss, G. A., Winkler, P. F., \& Canizares, C. R. 1982, ApJ, 258, 22

Reynoso, E. M., Dubner, G. M., Goss, W. M., \& Arnal, E. M. 1995, AJ, 110, 318

Reynoso, E. M., Green, A. J., Johnston, S., et al. 2003, MNRAS, 345, 671

Reynoso, E. M., Moffett, D. A., Goss, W. M., et al. 1997, ApJ, 491, 816

Rishbeth, H. 1958, Aust. J. Phys., 11, 550

Safi-Harb, S., \& Ögelman, H. 1997, ApJ, 483, 868

Teske, R. G., \& Petre, R. 1987, ApJ, 314, 673

Winkler, P. F., \& Kirshner, R. P. 1985, ApJ, 299, 981

Winkler, P. F., Turtle, J. H., Kirshner, R. P., \& Irwin, M. J. 1988, in Supernova Remnants and the Interstellar Medium, ed. R. S. Roger, \& T. Landecker (Cambridge: Cambridge Univ. Press), 65

Woermann, B., Gaylard, M. J., \& Otrupcek, R. 2000, MNRAS, 317, 421 